\title{
Balancing Between Power Optimization and Iub Efficiency in MBMS Enabled UMTS Networks
}

\author{
Antonios Alexiou ${ }^{1,2}$, Christos Bouras ${ }^{1,2}$ and Vasileios Kokkinos ${ }^{1,2}$ \\ ${ }^{1}$ Computer Engineering and Informatics Dept., Univ. of Patras, Greece \\ ${ }^{2}$ Research Academic Computer Technology Institute, Greece \\ University of Patras, Rio Campus, 26500 Rio, Patras, Greece \\ alexiua@cti.gr,bouras@cti.gr, kokkinos@cti.gr
}

\begin{abstract}
Multicast transmission is one of the major goals for Universal Mobile Telecommunication System (UMTS) that motivated the $3^{\text {rd }}$ Generation Partnership Project (3GPP) to launch the Multimedia Broadcast/Multicast Service (MBMS) framework. MBMS is a key framework that constitutes a significant step towards the so-called Mobile Broadband by efficiently utilizing network and radio resources, both in the core network and more importantly, in the UMTS Terrestrial Radio Access Network (UTRAN). One of most important problems that MBMS is currently facing is the assignment of the appropriate transport channel for the transmission of MBMS data. This paper proposes an alternative solution to the problem of efficient transport channel selection. In particular, we introduce a costbased scheme for the efficient radio bearer selection that minimizes the delivery cost of the multicast data. In our approach, the telecommunication cost over the most crucial interfaces of UMTS is calculated and based on this cost, the appropriate radio bearer is selected.
\end{abstract}

\section{Introduction}

One of the key aspects of MBMS defined in the 3GPP specifications is power control, since the available power of the base station is limited [1]. Another important challenge for MBMS is the need for optimization of data transmission over the UTRAN interfaces. Efficient mechanisms in MBMS should deal with a major aspect of MBMS traffic. This aspect is the selection of the appropriate transport channel for the transmission of MBMS traffic to multicast users after calculating the power requirements of each channel and the traffic load over the UTRAN interfaces.

Current approaches indicate that MBMS traffic can be provided in each cell by either multiple Point-to-Point (PTP) channels or by a single Point-to-Multipoint (PTM) channel. MBMS specifications deal with this issue with the introduction of MBMS Counting Mechanism of UMTS [2]. According to this mechanism, the 
decision on the threshold between PTP and PTM bearers is operator dependent, although it is proposed that it should be based on the number of serving MBMS users. Nevertheless, the MBMS Counting Mechanism provides a non realistic approach because mobility and current location of the mobile users are not taken into account; while on the other hand the base station's transmission power is not considered.

The inefficiencies of the MBMS Counting Mechanism motivated novel approaches, indicating that the assignment of the radio bearer should be performed in order to minimize the power requirements of the base stations [3]. A study under these assumptions is presented in [1]. However, this work considers only the air interface of the UMTS network and does not take into account other crucial interfaces of the UMTS network. Moreover, this work does not consider the enhancements that High Speed Downlink Packet Access (HSDPA) technology can provide by increasing data rates and decreasing power requirements, which however are considered in [4].

The fact that the Iub interface capacity is limited, stresses the need for considering this interface when selecting the most efficient radio bearer for the transmission of the MBMS data. Under this prism, in this paper we analytically present the multicast mode of MBMS and propose a novel approach that calculates the cost over the most crucial interfaces of the UMTS network during MBMS transmissions; and based on this calculation, allows the efficient transport channel assignment. The goal achieved by this work is twofold. At a first level, due to the fact that the MBMS Counting Mechanism is an open issue for 3GPP, our approach constitutes a more realistic and adaptive to dynamic wireless environments approach, by employing a cost-based switching criterion when selecting transport channel for MBMS transmissions. At a second level, our approach contributes to Radio Resource Management mechanism of UMTS by presenting a novel framework for MBMS that optimally utilizes power and network resources.

The paper is structured as follows: Section 2 provides an overview of the UMTS and MBMS architecture. Section 3 presents a cost analysis method for the evaluation of the MBMS multicast mode; while Section 4 examines the results of the analysis. Finally, some concluding remarks and planned next steps are described in Section 5.

\section{UMTS and MBMS Architecture}

A UMTS network consists of two land-based network segments: the Core Network (CN) and the UTRAN (Fig. 1). The $\mathrm{CN}$ is responsible for switching/routing voice and data connections, while the UTRAN handles all radio-related functionalities. The $\mathrm{CN}$ consists of two service domains: the CircuitSwitched (CS) and the Packet-Switched (PS) service domain. The PS portion of the CN in UMTS consists of two kinds of General Packet Radio Service (GPRS) Support Nodes (GSNs), namely Gateway GSN (GGSN) and Serving GSN 
(SGSN). An SGSN is connected to GGSN via the Gn interface and to UTRAN via the Iu interface. UTRAN consists of the Radio Network Controller (RNC) and the Node B. Node B constitutes the base station and provides radio coverage to one or more cells. Node B is connected to the User Equipment (UE) via the Uu interface and to the RNC via the Iub interface [5].

3GPP is currently standardizing MBMS. The major modification compared to the GPRS platform is the addition of a new entity called Broadcast Multicast Service Center (BM-SC) (Fig. 1). The BM-SC communicates with the existing UMTS/GSM networks and external Public Data Networks [6].

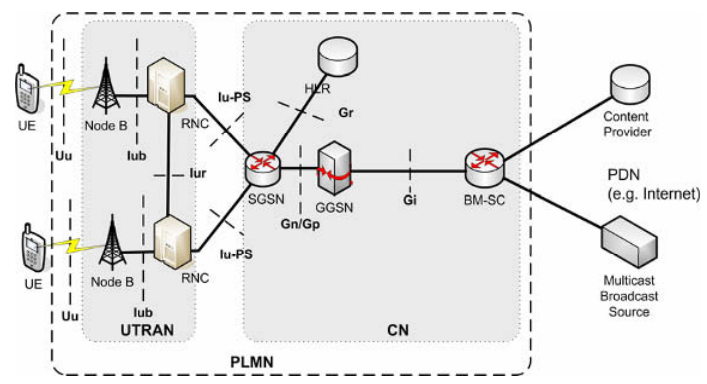

Fig. 1. UMTS and MBMS architecture

Regarding the transmission of the MBMS packets over the UTRAN interfaces, it may be performed on common (Forward Access Channel - FACH), on dedicated (Dedicated Channel - DCH) or on shared channels (High SpeedDownlink Shared Channel - HS-DSCH) [5], [7].

\section{Cost Analysis of the MBMS Multicast Mode in UTRAN}

As performance metric for our analysis, we consider the transmission cost for packet delivery only over the UTRAN interfaces and not the delivery cost over the interfaces of the $\mathrm{CN}$. In order to justify our choice, we briefly summarize the five steps occurred for the delivery of the multicast packets to the users of the multicast group.

With multicast, the packets are forwarded only to those Node Bs that have multicast users. Firstly, the BM-SC receives a multicast packet and forwards it to the GGSN that has registered to receive the multicast traffic. Secondly, GGSN receives the multicast packet and by querying its multicast routing lists, it determines which SGSCs have multicast users residing in their respective service areas. Thirdly, the destination SGSNs receive the multicast packets; and having queried their multicast routing lists, determine which RNCs should receive the multicast packets. The process of multicast packet delivery continues with the transmission of the multicast data over the UTRAN interfaces. Once the destination RNCs receive the multicast packet, they send it to the Node Bs that have established the appropriate radio bearers for the multicast data transmission. 
Finally, the users receive the packets on the appropriate radio bearers either by PTP channels, transmitted to individual users separately, or by PTM channels, transmitted to all members in the cell [8].

At this point, we have to mention that the process of packets' transmission in the $\mathrm{CN}$ is independent of the transport channel that is assigned for the transmission of the multicast data over the UTRAN interfaces. This in turn means that the cost for the transmission of multicast packets from BM-SC to RNC is independent of the transport channel that will be assigned in UTRAN. On the other hand, the packet delivery cost over UTRAN interfaces strongly depends on the transport channel selected for the transmission of multicast traffic to mobile users. The analysis in the rest of this paper will focus on the packet delivery cost over the UTRAN interfaces.

The telecommunication cost [9] of multicast packet transmission over the UTRAN interfaces $\left(C_{U T R A N}\right)$ is derived from the following equation [10]:

$$
C_{\text {UTRAN }}=\left\{\begin{array}{c}
c_{i}\left(D_{r b}+p_{b}\right)+c_{w}\left(D_{F A C H}\right), \text { if channel }=F A C H \\
c_{i}\left(N_{U E} \cdot\left(D_{r b}+p_{b}\right)\right)+c_{w}\left(\sum_{i=1}^{N_{U E}} D_{D C H}(i)\right), \text { if } \quad \text { channel }=D C H \\
c_{i}\left(N_{U E} / 2 \cdot\left(D_{r b}+p_{b}\right)\right)+c_{w}\left(D_{H S-D S C H}\right), \text { if } \quad \text { channel }=H S-D S C H
\end{array}\right\}=c_{i} \cdot D_{l u b}+c_{w} \cdot D_{U u}
$$

where $N_{U E}$ represents the total number of multicast users in the cell, $D_{r b}$ is the transmission cost of packet delivery over the Iub interface (RNC - Node B), $p_{b}$ is the processing cost of multicast packet delivery at Node $\mathrm{B}, D_{D C H}, D_{F A C H}, D_{H S-D S C H}$ are the transmission costs of packet delivery over the $\mathrm{Uu}$ interface with $\mathrm{DCH}$, FACH and HS-DSCH respectively and $c_{i}$ and $c_{w}$ are the weights of Iub and Uu respectively (where $c_{i}+c_{w}=1$ ). According to equation (1) the total telecommunication cost of every channel consists of the transmission cost over the Iub interface plus the cost over the Uu interface, after multiplying each cost with an appropriate weight.

Parameter $C_{\text {UTRAN }}$ is calculated for each cell and for each transport channel in a cell separately and these calculations are performed in the RNC. Based on these calculations, the RNC selects for the transmission of the MBMS data, the transport channel that ensures the lowest cost over the UTRAN interfaces (i.e. the transport channel that generates the smallest value for parameter $\left.C_{U T R A N}\right)$.

\subsection{Cost over the Iub Interface}

One of the significant operational expenses in UMTS networks is the Iub transmission between the RNC and Node Bs. The fact that the capacity of the Iub interface is limited indicates that this interface should be included in our analysis. In general, PTM transmissions bring benefit in Iub capacity compared to PTP transmissions. In PTP transmissions a dedicated Iub capacity for each user is 
required, whereas in PTM transmissions only a single Iub capacity allocation is required. The Iub allocation is performed per base station, according to Release 7 3GPP specifications [5].

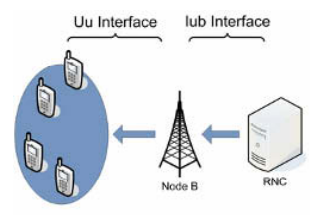

(a)

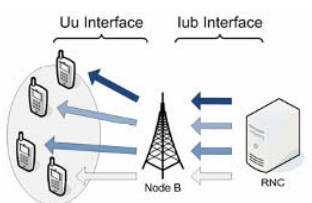

(b)

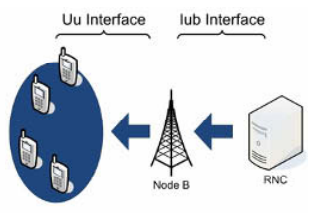

(c)

Fig. 2. MBMS transmissions over Iub and Uu with: (a) FACH, (b) DCH, (c) HS-DSCH

Regarding the cost over the Iub interface, when FACH is employed as transport channel, each multicast packet is sent once over the Iub interface and then the packet is transmitted to the UEs that are served by the corresponding Node B (Fig. 2a). On the other hand, when DCHs are used for the transmission of the multicast packets, each packet is replicated over the Iub as many times as the number of multicast users that the corresponding Node B serves (Fig. 2b). In HSDPA, the Iub efficiency is improved by a factor of 2 compared to that of Wideband Code Division Multiple Access (WCDMA) (Fig. 2c). This is a significant improvement, which basically means that twice as many user bits can be transmitted through the same Iub with HSDPA compared to Release 99 WCDMA. This improvement mainly comes from fast dynamic sharing of the HSDPA Iub bandwidth allocated between active users [7].

Fig. 2 depicts that PTM transmissions bring benefit in Iub capacity, while on the other hand DCH has increased Iub capacity requirements compared to HSDSCH in PTP transmissions. As the Iub capacity is limited, Fig. 2 indicates that the packet delivery cost over the Iub interface should also be taken into account when selecting the most efficient radio bearer for the transmission of the MBMS data.

As far as equation (1) is concerned, as presented and analyzed in our previous work [10], the packet transmission cost in any segment of the UMTS network depends on two parameters: the number of hops between the edge nodes of this network segment and the capacity of the link of the network segment. This means that $D_{r b}=l_{r b} / k_{r b}$, where $k_{r b}$ represents the capacity factor of the link between the RNC and Node B and $l_{r b}$ the hops between these two nodes. Moreover, for the cost analysis and without loss of generality, we assume that the distance between the RNC and Node B is 1 hop and the capacity factor of this link is 0.5 . Therefore, the value for parameter $D_{r b}$ is set to 2 . We have also estimated the value of parameter $p_{b}$ that represents the processing cost of packet delivery at Node B. This value is set to 1 , as in [10]. 


\subsection{Cost over the Uu Interface}

According to equation (1), $C_{U T R A N}$ depends firstly on the population of the multicast group within a cell and secondly on the transport channel that is used. $D_{D C H}, D_{F A C H}$ and $D_{H S-D S C H}$ represent the cost over the Uu interface. More specifically, $D_{D C H}$, represents the cost of using a single DCH to transmit the multicast data to a single multicast user of the network, $D_{F A C H}$ represents the cost of using a FACH channel to serve all multicast users residing in a specific cell, while $D_{H S-D S C H}$ represents the cost of using a HS-DSCH that is shared to the multicast users residing in a specific cell.

In order to calculate the costs over the Uu interface, we will follow an approach that reflects the most recent specifications and requirements of 3GPP to consider the power consumption during MBMS transmissions [3]. Therefore, in our analysis, the fundamental factor that determines the transmission cost over the Uu interface is the amount of Node B's transmission power when using HS-DSCH, $\mathrm{DCH}$ or FACH transport channels. To this direction, we will first examine the power profile of each transport channel; and then, we will define the exact cost introduced by Uu interface during MBMS multicast transmission.

\subsubsection{HS-DSCH Power Profile}

HS-DSCH is a rate controlled rather than a power controlled transport channel. Although there are two basic modes for allocating HS-DSCH transmission power [7], in this paper we will focus on a dynamic method in order to provide only the required, marginal amount of power so as to satisfy all the multicast users. Two major measures for HSDPA power planning are: the HS-DSCH Signal-toInterference-plus-Noise Ratio (SINR) metric and the Geometry factor $(G)$. SINR for a single-antenna Rake receiver is calculated as in (2):

$$
S I N R=S F_{16} \frac{P_{H S-D S C H}}{p P_{\text {own }}+P_{\text {other }}+P_{\text {noise }}}
$$

where $P_{H S-D S C H}$ is the HS-DSCH transmission power, $P_{\text {own }}$ is the own cell interference experienced by the mobile user, $P_{\text {other }}$ the interference from neighboring cells and $P_{\text {noise }}$ the Additive White Gaussian Noise. Parameter $p$ is the orthogonality factor ( $p=0$ : perfect orthogonality), while $S F_{16}$ is the spreading factor of 16 .

There is a strong relationship between the HS-DSCH allocated power and the obtained MBMS cell throughput. This relationship can be disclosed in the three following steps. Initially, we have to define the target MBMS cell throughput. Once the target cell throughput is set, the next step is to define the way that this throughput relates to the SINR. Finally, we can describe how the required HSDSCH transmission power $\left(P_{H S-D S C H}\right)$ can be expressed as a function of the SINR value and the user location (in terms of $G$ ) as in equation (3) [7]: 


$$
P_{H S-D S C H} \geq \operatorname{SINR}\left[p-G^{-1}\right] \frac{P_{o w n}}{S F_{16}}
$$

\subsubsection{DCH Power Profile}

The total downlink transmission power allocated for all MBMS users in a cell that are served by multiple DCHs is variable. It mainly depends on the number of serving users, their distance from the base station, the bit rate of the MBMS session and the experienced signal quality, $E_{b} / N_{0}$, for each user. Equation (4) calculates the total DCH power required for the transmission of the data to $n$ users in a specific cell [11].

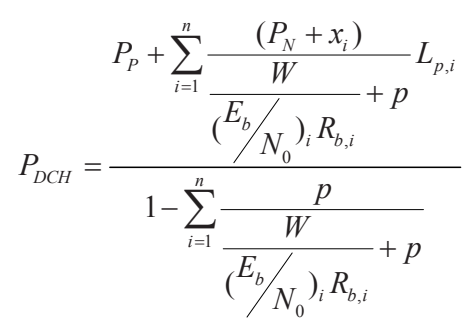

where $P_{D C H}$ is the base station's total transmitted power, $P_{P}$ is the power devoted to common control channels, $L_{p, i}$ is the path loss, $R_{b, i}$ the $i^{\text {th }}$ user transmission rate, $W$ the bandwidth, $P_{N}$ the background noise, $p$ is the orthogonality factor and $x_{i}$ is the interference observed by the $i^{\text {th }}$ user given as a function of the transmitted power by the neighboring cells $P_{T j}, j=1, \ldots K$ and the path loss from this user to the $j^{\text {th }}$ cell $L_{i j}$.

\subsubsection{FACH Power Profile}

A FACH essentially transmits at a fixed power level since fast power control is not supported in this channel. FACH is a PTM channel and must be received by all users throughout the part of the cell that the users are found. Table 1 presents the FACH transmission power levels obtained for various cell coverage areas [12]. Depending on the distance between the user with the worst path loss and the Node $\mathrm{B}$, the RNC adjusts the FACH transmission power in one of the ten levels presented in Table 1, so as to ensure a reliable reception of the MBMS data. The FACH transmission power levels presented in Table 1 correspond to the case of a 64 Kbps MBMS service, where no Space Time Transmit Diversity (STTD) is assumed. In addition, Transmission Time Interval (TTI) is set to $80 \mathrm{~ms}$ and Block Error Rate (BLER) target is 1\% [12]. 
Table 1. FACH Tx power levels

\begin{tabular}{ll}
\hline Cell coverage $(\%)$ & Required Tx power $(\mathrm{W})$ \\
\hline 10 & 1.4 \\
20 & 1.6 \\
30 & 1.8 \\
40 & 2.0 \\
50 & 2.5 \\
60 & 3.0 \\
70 & 3.6 \\
80 & 4.8 \\
90 & 6.4 \\
100 & 12.0 \\
\hline
\end{tabular}

\subsubsection{Calculation of the Cost over the Uu Interface}

In this section we will present the procedure for calculating the $D_{D C H}, D_{F A C H}$ and $D_{H S-D S C H}$ costs. The mechanism for this procedure, the block diagram of which is illustrated in Fig. 3, runs at the RNC. According to Fig. 3, the procedure can be divided into three operation phases. These are: the parameter retrieval phase, the cost calculation phase and the event scheduling phase.

The parameter retrieval phase is responsible for retrieving the parameters of the existing MBMS users (through uplink channels) in each cell. These parameters are the distance of each UE from the Node B and the $E_{b} / N_{0}$ (or SINR for the HS$\mathrm{DSCH}$ case) requirement per UE. In order to retrieve this information, the RNC broadcasts a message to the UEs belonging to a specific MBMS group and each user of the group responds to this message by indicating its location and its experienced signal quality. The MBMS bit rate service is assumed to be already known (in the BM-SC).

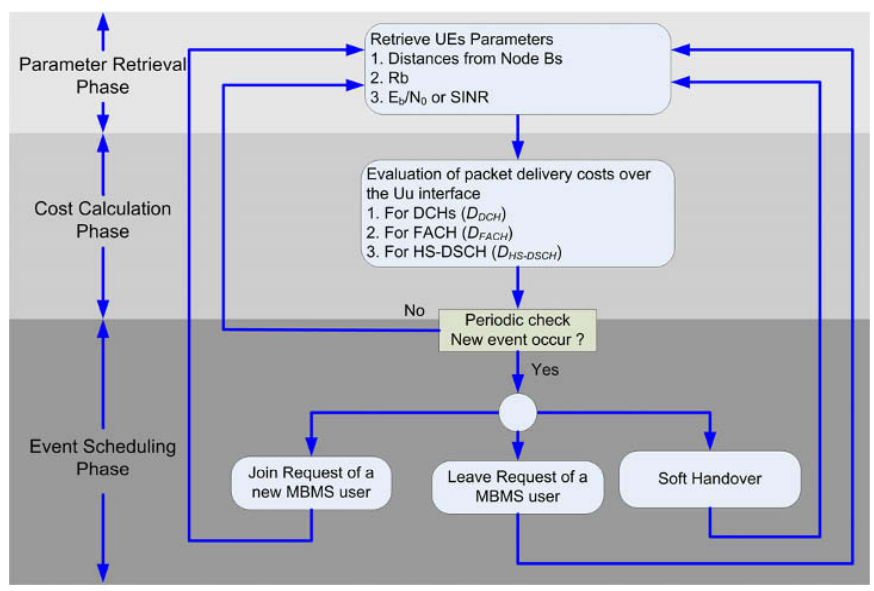

Fig. 3. Calculation of packet delivery cost over the Uu interface 
The cost calculation phase substantially processes the data received from the parameter retrieval phase. During this phase, the required cost to be allocated for each cell is computed. The computation is based on the assumption that the transmission of the multicast data over the UTRAN interfaces can be performed with:

- Multiple DCHs (DCHs case).

- FACH with such power to serve the worst path loss user (FACH Dynamic case).

- HS-DSCH (HS-DSCH case).

In other words, the telecommunication cost is computed, assuming that all UEs in a cell could be served with the above three possible ways. For the DCHs case, the computation takes into account the parameters defined in the parameter retrieval phase and calculates the required cost $\left(D_{D C H}\right)$ as in equation (4). For the FACH Dynamic case, the cost $\left(D_{F A C H}\right)$ is computed depending on the user with the worst path loss and according to Table 1, as described in section 3.2.3. Finally, for the HS-DSCH case, the cost $\left(D_{H S-D S C H}\right)$ is computed as in equation (3).

The algorithm enters the event scheduling phase, only if one of the following three different events occurs during a MBMS session: a join request from a new MBMS user, a leave request from an existing MBMS user or a handover. The algorithm handles these three events with the absolutely same way, since the parameters of all the users are updated in regular time intervals.

The above description refers to a dynamic model, in the sense that the UEs are assumed to be moving throughout the topology. The parameter retrieval phase is triggered at regular time intervals so as to take into account the users' mobility and the three events of the event scheduling phase. Therefore, the $D_{D C H}, D_{F A C H}$ and $D_{H S-D S C H}$ costs must be computed periodically at a predetermined frequency rate. This periodic computation inserts a further complexity for RNC, as this information is carried in uplink channels. Moreover, a certain bandwidth fraction must be allocated for the transmission of this information in the uplink channel, thus resulting to a capacity reduction.

\section{Performance Evaluation}

This section presents analytical simulation results for the performance evaluation of our approach. The main assumptions that were used in our simulations are presented in Table 2 and refer to a macro cell environment [5], [13]. In addition, no STTD is assumed, while BLER target is set to $1 \%$.

Our goal is to demonstrate and highlight the advantages of our approach through a mathematical analysis, which however totally simulates the macro cell environment. To this direction, we examine certain scenarios indicative of the way that our mechanism works and how the appropriate channels are assigned.

One point that needs attention is that the total cost computed from equation (1) is a positive number, which however does not have units. According to equation 
(1), each channel is associated with a cost and by comparing the corresponding costs, the mechanism assigns the most appropriate transport channel.

Table 2. Simulation assumptions

\begin{tabular}{ll}
\hline Parameter & Value \\
\hline Cellular layout & 18 hexagonal grid cells \\
Sectorization & 3 sectors $/$ cell \\
Site-to-site distance & $1 \mathrm{Km}$ \\
Maximum BS Tx power & $20 \mathrm{~W}(43 \mathrm{dBm})$ \\
Other BS Tx power & $5 \mathrm{~W}(37 \mathrm{dBm})$ \\
CPICH power & $2 \mathrm{~W}$ \\
Common channel power & $1 \mathrm{~W}(30 \mathrm{dBm})$ \\
Propagation model & Okumura Hata \\
Multipath channel & Vehicular A $(3 \mathrm{~km} / \mathrm{h})$ \\
Orthogonality factor & 0.5 \\
$E_{b} / N_{0}$ target & $5 \mathrm{~dB}$ \\
\hline
\end{tabular}

\subsection{Scenario 1: Balancing between Power Optimization and Iub Efficiency}

This section describes the role of weights $c_{i}$ and $c_{w}$ in equation (1). These weights define which UTRAN interface (Iub or $\mathrm{Uu}$ ) plays a more significant role in the calculation of the $C_{U T R A N}$. In general, high values for weight $c_{i}$ indicate that the transport channel selection is determined based mainly on the cost over Iub interface.

Fig. 4 presents how the total cost for the transmission of a $64 \mathrm{Kbps}$ MBMS service is changing as the $c_{w}$ increases from 0 to $100 \%$. In the particular scenario, a group of 6 users located at a fixed distance from Node B (35\% cell coverage) receive the MBMS service. The small number of users and the relatively small area that needs to be covered regularly would have favored the use of multiple DCHs. However, as it appears from Fig. 4 for small weights of wireless channel, DCH has the highest cost. This occurs because even if the use of multiple DCHs has lower power requirements than $\mathrm{FACH}$ or $\mathrm{HS}-\mathrm{DSCH}$ for the specific user distribution, the small value of $c_{w}$ (high value of $c_{i}$ ) makes the cost for packet replication over Iub the dominant term in equation (1). As the weight increases (transmission power plays a more important role), the cost for $\mathrm{DCH}$ remains lower than the cost of FACH and HS-DSCH. In the following scenarios, the value of $c_{w}$ is set to 0.9. This high value of $c_{w}$ is selected to reflect the fact that power is a limited resource and in parallel, to meet the $3 \mathrm{GPP}$ requirements for reducing power requirements during MBMS transmissions. 


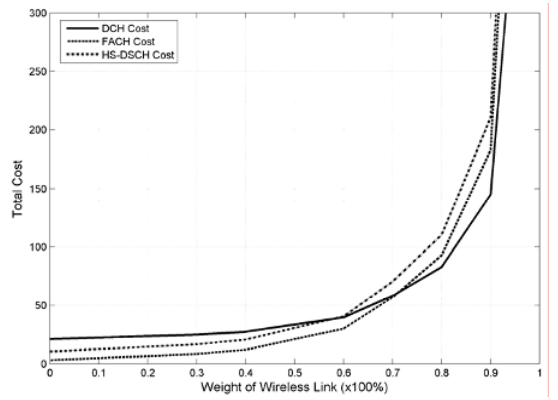

Fig. 4. Costs vs. Weight of wireless link

\subsection{Scenario 2: Join and Leave Requests}

The second scenario examines how the join and leave requests by MBMS users are handled by the mechanism. According to this scenario, the UEs appear in random initial positions and then move randomly throughout the cell, while the number of serving users varies during the simulation. More specifically, the number of users that receive a $64 \mathrm{Kbps}$ MBMS service initially increases, reaching 35 UEs at simulation time $175 \mathrm{sec}$. For the following 80 seconds, the number of users remains constant. From simulation time $255 \mathrm{sec}$ the number of users is decreasing and finally, at the end of the simulation only 6 UEs receive the 64 Kbps MBMS service (Fig. 5).

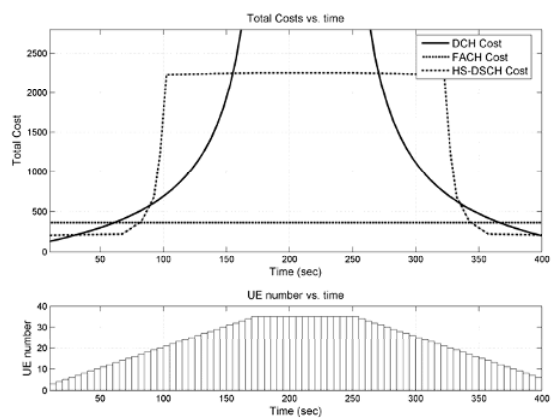

Fig. 5. Costs vs. Time for successive join and leave requests

The algorithm presented in the previous sections that runs at the RNC will force the Node B to select, at each instant, the channel with the lowest telecommunication cost over the UTRAN interfaces. Thus, in the beginning of the simulation when the number of UEs is small, the most efficient channel is the DCH. The increase in the number of UEs causes a switch from DCHs to HS$\mathrm{DSCH}$ at simulation time $30 \mathrm{sec}$ (when the UE population is 7). An additional increase in the number of UEs results to a switch from HS-DSCH to a single 
FACH (at simulation time $80 \mathrm{sec}$ when the UE population is 17), with transmission power high enough to cover the UE with the worst path loss. A further increase in the UE number does not involve any change. The decrease in the number of UEs causes the exact opposite results.

\subsection{Scenario 3: Handover}

This scenario presents the operation of the mechanism during handover. Each Node B in the topology has to serve a number of randomly moving UEs with initial positions as in Fig. 6. However, Node B1 initially serves eight UEs, four of which will follow a predefined route so that handover will take place. More specifically, as shown in Fig. 6, UE1 and UE2 will move towards Node B2, while UE3 and UE4 towards Node B3.

Fig. 7 depicts the telecommunication cost over the UTRAN interfaces for the Node Bs under study when DCH, FACH and HS-DSCH are used. The three Node Bs under study, in other words, the three Node Bs that participate in the process of handover are: Node B1, Node B2 and Node B3. The algorithm running at the RNC will command each Node B to select, at each instant, the transport channel that inserts the lowest telecommunication cost.

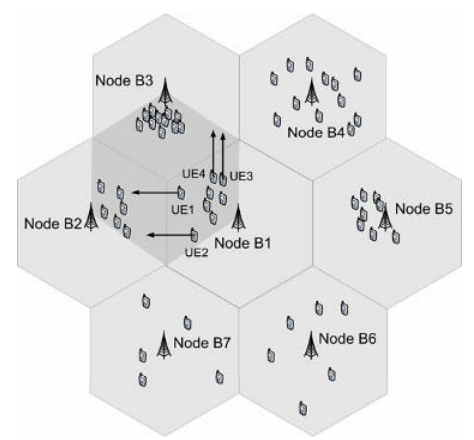

Fig. 6. UEs' initial locations and routes

The small UE population in Node B1 favors the deployment of DCHs. Totally, 4 UEs leave its area and this is obvious by the four abrupt decrements in DCHs cost (Fig. 7a). The FACH and HS-DSCH costs remain high, until the 4 UEs leave the cell (simulation time $255 \mathrm{sec}$ ), when an abrupt decrement in their costs is observed. This happens because after the UEs leave the cell, the FACH and HSDSCH will only have to serve UEs close to Node B1 (Fig. 6). Nevertheless, even if the 4 UEs leave the cell, multiple DCHs should be deployed as the cost in this case remains lower (Fig. 7a). 

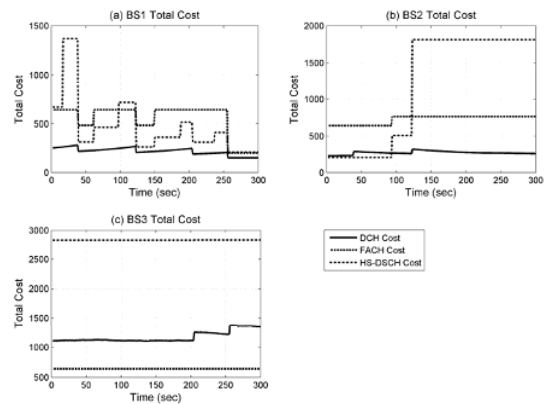

Fig. 7. Costs vs. Time during handover

On the other hand, a decrement in Node B1's costs is followed by a simultaneous increment in another Node B's costs. For example, at simulation time $122 \mathrm{sec}$, UE2 leaves the coverage area of Node B1 and enters the coverage area of Node B2 (Fig. 6). The DCH and HS-DSCH costs in Fig. 7b increase because in both cases, Node B2 will have to employ one more PTP connection for the new user.

It is worth mentioning, that during the simulations the appropriate channel was selected independently of the UE number and location. The corresponding costs over the UTRAN interfaces were compared and the channel with the lowest cost was selected. This fact makes our approach more powerful and more resistant in changes.

\section{Conclusions and Future Work}

In this paper, we presented an overview of the MBMS multicast mode and motivated by the fact that the Iub capacity is limited, we introduced a power/cost based scheme for the efficient radio bearer selection that: firstly, minimizes the delivery cost of the multicast data over the UTRAN interfaces and secondly, reduces the power requirements during MBMS transmissions. Our approach considers all the basic functionalities of the two 3GPP approaches (TS 25.346 [2] and TR 25.922 [3]) and incorporates several enhancements. Contrary to TS 25.346, our approach considers users' mobility and utilizes a dynamic scheme for switching between channels. Contrary to TR 25.922, both PTP channels (HSDSCH and DCH) are supported. Finally, contrary to TS 25.346 and TR 25.922, our approach supports FACH dynamic power allocation in order to reduce power consumption during PTM transmissions.

The step that follows this work is to expand our approach in order to provide an efficient session assignment scheme when multiple MBMS sessions run simultaneously in the network. At a second level we plan to study the complexity that the mechanism inserts in RNCs due to its dynamic and periodic nature. 


\section{References}

1. Alexiou, A., Bouras, C., Rekkas, E.: A Power Control Scheme for Efficient Radio Bearer Selection in MBMS. The 8th IEEE 21st International Symposium on World of Wireless, Mobile and Multimedia Networks (WoWMoM) (2007)

2. 3GPP, TS 25.346 V8.1.0. Introduction of the Multimedia Broadcast Multicast Service (MBMS) in the Radio Access Network (RAN); Stage 2, (Release 8)

3. 3GPP, TR 25.922 V7.1.0. Technical Specification Group Radio Access Network; Radio resource management strategies (Release 7)

4. Alexiou, A., Bouras, C., Kokkinos, V., Rekkas, E.: Efficient Assignment of Multiple MBMS Sessions in B3G Networks. 2008 IEEE 68th Vehicular Technology Conference (VTC2008 Fall) (to appear)

5. Holma, H., Toskala, A.: WCDMA for UMTS: HSPA Evolution and LTE. 4th edition, John Wiley \& Sons (2007)

6. 3GPP, TS 22.146 V8.3.0. Technical Specification Group Services and System Aspects; Multimedia Broadcast/Multicast Service; Stage 1 (Release 8)

7. Holma, H., Toskala, A.: HSDPA/HSUPA for UMTS: High Speed Radio Access for Mobile Communications. John Wiley \& Sons (2006)

8. Alexiou, A., Antonellis, D., Bouras, C., Papazois, A.: An Efficient Multicast Packet Delivery Scheme for UMTS. The 9th ACM/IEEE International Symposium on Modeling, Analysis and Simulation of Wireless and Mobile Systems (MSWiM 2006) pp. 147-150

9. Ho, J., Akyildiz, I.: Local anchor scheme for reducing signaling costs in personal communications networks. IEEE/ACM Transactions on Networking, Vol. 4, No. 5 (1996) pp. 709-725

10. Alexiou, A., Bouras, C.: Multicast in UMTS: Evaluation and Recommendations. Wireless Communications and Mobile Computing Journal. Wiley InterScience (2006) (in press)

11. Perez-Romero, J., Sallent, O., Agusti, R., Diaz-Guerra, M.: Radio Resource Management Strategies in UMTS. John Wiley \& Sons (2005)

12. 3GPP, TS 25.803 V6.0.0. S-CCPCH performance for MBMS (Release 6)

13. 3GPP, TR 101.102 V3.2.0. Universal Mobile Telecommunications System (UMTS); Selection procedures for the choice of radio transmission technologies of the UMTS (UMTS 30.03 version 3.2.0) 